University of Nebraska - Lincoln

DigitalCommons@University of Nebraska - Lincoln

Norman R. Simon Papers

Research Papers in Physics and Astronomy

7-20-1993

\title{
THE RR LYRAE VARIABLES IN THE GLOBULAR CLUSTER M68
}

Christine M. Clement

University of Toronto, cdement@doncarlo.astro.utoronto.ca

Stephen Ferance

University of Toronto

Norman R. Simon

University of Nebraska - Lincoln, nsimon@unl.edu

Follow this and additional works at: https://digitalcommons.unl.edu/physicssimon

Clement, Christine M.; Ferance, Stephen; and Simon, Norman R., "THE RR LYRAE VARIABLES IN THE GLOBULAR CLUSTER M68" (1993). Norman R. Simon Papers. 41.

https://digitalcommons.unl.edu/physicssimon/41

This Article is brought to you for free and open access by the Research Papers in Physics and Astronomy at DigitalCommons@University of Nebraska - Lincoln. It has been accepted for inclusion in Norman R. Simon Papers by an authorized administrator of DigitalCommons@University of Nebraska - Lincoln. 


\title{
THE RR LYRAE VARIABLES IN THE GLOBULAR CLUSTER M68
}

\author{
Christine M. Clement and Stephen Ferance \\ David Dunlap Observatory, Department of Astronomy, University of Toronto, Toronto, Ontario, Canada, M5S 1A1 \\ AND \\ NoRMAN R. SimON \\ Department of Physics and Astronomy, University of Nebraska, Lincoln, NE 68588 \\ Received 1992 September 10; accepted 1993 January 29
}

\begin{abstract}
New observations, made with the Helen Sawyer Hogg telescope at Las Campanas, have been analyzed in a search for double-mode pulsators (RRd stars) in the metal-poor globular cluster, Messier 68 . Of the 30 stars studied, nine have been identified as RRd stars; V33, which exhibited the characteristics of an RRd star in 1950, now appears to be an RRc star. Reliable periods and period ratios have been determined for six of the RRd stars. Masses for these RRd stars, calculated from fitting formulas given by Kovács, Buchler, \& Marom, range from 0.75 to $0.90 M_{\odot}$, depending on the assumed luminosity and metal abundance. These masses are in the same range as those for the RRd stars in M15, whose RRd sample resembles that of M68 very closely. Fourier parameters determined for the light curves of the M68 variables show that the RRc stars in the two clusters are also very similar. In particular, on the plot of phase parameter $\phi_{31}$ with period, the M15 and M68 RRc samples are virtually indistinguishable. A comparison of the new M68 observations with observations made $40 \mathrm{yr}$ ago shows that the periods of some of the stars have changed, but the $40 \mathrm{yr}$ interval is too short for detecting period changes caused by evolutionary effects.

Subject headings: globular clusters: individual (M68) - stars: variables: other (RR Lyrae) - stars: oscillations
\end{abstract}

\section{INTRODUCTION}

M68 is a metal-poor Oosterhoff type II globular cluster with $[\mathrm{Fe} / \mathrm{H}]=-2.09$ according to Suntzeff, Kinman, \& Kraft (1991). It has 37 known RR Lyrae variables, and among these are a number of double-mode (RRd) pulsators (Andrews 1979, 1980; Clement 1990). In the previous work on M68, reliable periods were determined for only one of the RRd stars, while for the others, there was some uncertainty, particularly for the secondary periods. Therefore, an observing program to determine periods for the RRd stars in M68 was undertaken.

\section{THE OBSERVATIONAL DATA}

The present investigation is based on observations made during the years 1986 to 1991 with the $61 \mathrm{~cm}$ Helen Sawyer Hogg telescope (plate scale 22.6 arcsec $\mathrm{mm}^{-1}$ ) of the University of Toronto Southern Observatory, at the Las Campanas Observatory of the Carnegie Institution of Washington. In total, $157 \mathrm{~B}$ photographs $(103 \mathrm{aO}+\mathrm{GG} 385)$ were taken and the exposure times ranged from 20 minutes, when the cluster was near the meridian, to 40 minutes at large hour angles $\left(4^{\mathrm{h}}\right)$. The observations are summarized in Table 1 which lists the year, the month, the number of nights in the month that observations were made, and the number of plates taken that month. The important thing to note in Table 1 is that the 1989 observations were made over an interval of several months so that the number of cycles that elapsed in $1 \mathrm{yr}$ could be precisely determined. In a project to determine precise periods of $R R$ Lyrae variables, it is important to have observations made in different seasons of the year. Otherwise, the periods may be uncertain by 0.001 days, even when the observations span several years.

The plates were measured on a Cuffey iris astrophotometer. However, only 30 of the 37 RR Lyrae variables were measured because the other seven were in crowded regions near the cluster center and could not be adequately resolved. For a standard sequence, we selected and measured 21 stars, in the appropriate magnitude range, from the CCD photometry of M68 by McClure et al. (1987). In order to determine $B$ magnitudes for these standard stars on our photographic system, we fitted parabolae to the calibration curves (iris reading vs. magnitude for the standard stars) by the method of least squares. This analysis was carried out for 30 plates, and the results are shown in Table 2 . The star names $(\mathrm{A}-\mathrm{X})$ were designated in the present investigation, the $x, y$ coordinates are those given by McClure et al. (1987), and the CCD $B$ magnitudes were calculated from their values for $V$ and $B-V$. The photographic $B$ magnitudes are the mean values calculated from the 30 calibration curves, and the $\sigma$ values are their standard deviations. These photographic $B$ magnitudes were used for the reductions. A parabolic calibration curve was derived for each plate, and the $B$ magnitudes for the variable stars were calculated. These magnitudes, for the $30 \mathrm{RR}$ Lyrae stars that were measured, are listed in Table 3, along with the heliocentric Julian date at the mean time for each observation.

\section{CALCULATION OF THE PERIODS}

Two methods were used for determining the periods of the variable stars. First, a program which utilized the phasedispersion minimization (PDM) method of Stellingwerf (1978) was used. Then, to confirm the periods found by the PDM method, we used a program based on the Lomb-Scargle method (Lomb 1976; Scargle 1982). For increased efficiency, this latter program used Press \& Rybicki's (1989) algorithm for fast evaluation of the Lomb-Scargle statistic and Kurtz's (1985) recursion relation for computing the relevant sines and cosines at equispaced frequencies. Both methods gave the same primary periods for all of the stars. Once the primary periods were established, all of the stars were checked for the presence of a secondary period that might be caused by pulsation in 
TABLE 1

Las Campanas Observations of M68

\begin{tabular}{|c|c|c|c|}
\hline Year & Month & $\begin{array}{c}\text { Number of } \\
\text { Nights }\end{array}$ & $\begin{array}{c}\text { Total Number } \\
\text { of Plates }\end{array}$ \\
\hline $1986 \ldots \ldots \ldots \ldots \ldots \ldots \ldots$ & May & 10 & 32 \\
\hline \multirow[t]{2}{*}{$1987 \ldots \ldots \ldots \ldots \ldots \ldots$} & May & 5 & 11 \\
\hline & Jul & 2 & 3 \\
\hline $1988 \ldots \ldots \ldots \ldots \ldots \ldots \ldots$ & May & 6 & 15 \\
\hline \multirow{5}{*}{$1989 \ldots \ldots \ldots \ldots \ldots \ldots$} & Jan & 2 & 7 \\
\hline & Feb & 1 & 2 \\
\hline & Apr & 3 & 12 \\
\hline & Jun & 4 & 11 \\
\hline & Jul & 2 & 4 \\
\hline \multirow[t]{2}{*}{$1990 \ldots \ldots \ldots \ldots \ldots \ldots$} & Apr & 7 & 26 \\
\hline & May & 1 & 3 \\
\hline $1991 \ldots \ldots \ldots \ldots \ldots \ldots$ & May & 5 & 31 \\
\hline
\end{tabular}

another mode. In order to do this, we fitted a Fourier series,

$$
\operatorname{mag}=A_{0}+\sum_{j=1, n} A_{j} \cos \left(j \omega t+\phi_{j}\right),
$$

to the observed light curve for each star and the residuals (observed magnitude minus calculated magnitude) from these light curves were period searched. It is generally assumed that RR Lyrae stars which pulsate simultaneously in the fundamental and first overtone modes have period ratios $\left(\boldsymbol{P}_{\mathbf{1}} / \boldsymbol{P}_{\mathbf{0}}\right)$ between 0.73 and 0.76 (Cox, Hodson, \& Clancy 1983; Nemec 1985). Therefore, our search for secondary periods was limited to periods in the range that would give period ratios in this interval. Stars with primary periods less than 0.5 days were assumed to be pulsating in the first overtone mode and stars with primary periods greater than 0.5 days were assumed to be pulsating in the fundamental mode. Our analysis confirmed that variables $3,4,7,19,26,29$, and 31 are all double-mode pulsators as found in the previous investigation (Clement 1990). In addition, the RRd candidate stars, V21 and V34, were found to be RRd stars. However, for V33, which was suggested as a possible $\mathrm{RRd}$ candidate in the earlier study, no secondary oscillations were detected at a level higher than $0.07 \mathrm{mag}$, the standard deviation of the points about the light curve. None of the other stars was found to be a double-mode pulsator.

The earlier analysis of V33 was based on photographic observations made in 1950, and published by van Agt \& Oosterhoff (1959). They did not actually determine magnitudes, but instead, made visual estimates $(s)$ by means of an eyepiece and these estimates ranged in value from $s=0$ to 6 . All but two of the observations were made over an interval of 13 days so that secondary periods with a precision better than 0.002 days
TABLE 2

Sequence STARs for M68 ( $B$ MaG)

\begin{tabular}{|c|c|c|c|c|c|}
\hline Star & $x$ & $y$ & $B_{\mathrm{CCD}}$ & $B_{\mathrm{pg}}$ & $\sigma$ \\
\hline & 594.75 & 413.00 & 15.23 & 15.20 & 0.04 \\
\hline B. & 536.17 & 608.32 & 15.30 & 15.32 & 0.03 \\
\hline C . & 416.75 & 750.59 & 15.44 & 15.48 & 0.05 \\
\hline D . & 216.99 & 575.68 & 15.59 & 15.62 & 0.05 \\
\hline E......... & 594.47 & 716.90 & 15.80 & 15.82 & 0.04 \\
\hline$F \ldots \ldots$ & 281.14 & 49.79 & 15.95 & 15.99 & 0.02 \\
\hline & 673.68 & 408.16 & 15.98 & 15.91 & 0.05 \\
\hline H. & 605.42 & 565.61 & 15.99 & 15.96 & 0.03 \\
\hline $\mathbf{J}$. & 354.23 & 474.99 & 16.03 & 16.04 & 0.03 \\
\hline K & 496.36 & 452.43 & 16.18 & 16.15 & 0.04 \\
\hline L. & 679.06 & 489.51 & 16.19 & 16.17 & 0.05 \\
\hline $\mathbf{M}$. & 56.30 & 462.94 & 16.33 & 16.36 & 0.04 \\
\hline & 226.52 & 6.99 & 16.36 & 16.39 & 0.04 \\
\hline P.. & 487.89 & 569.90 & 16.44 & 16.41 & 0.04 \\
\hline Q & 17.50 & 369.91 & 16.53 & 16.55 & 0.05 \\
\hline $\mathbf{R}$ & 669.35 & 751.54 & 16.58 & 16.56 & 0.06 \\
\hline S. & 703.82 & 809.86 & 16.64 & 16.60 & 0.05 \\
\hline T. & 25.32 & 608.40 & 16.71 & 16.72 & 0.04 \\
\hline V & 222.33 & 149.62 & 16.73 & 16.77. & 0.03 \\
\hline & 34.05 & 151.78 & 16.83 & 16.85 & 0.04 \\
\hline X . & 330.95 & 92.98 & 17.00 & 16.94 & 0.05 \\
\hline
\end{tabular}

The $x, y$ coordinates are from McClure et al. 1987.

could not be determined. Nevertheless, there is some hint that pulsation in two modes was occurring in V33 in 1950. Period searches of these observations indicated a primary period of 0.3904 with amplitude $3.80(s)$ and $\sigma=0.58$ and a secondary period 0.5223 with amplitude 1.42 and $\sigma=0.57$. Thus, it is possible that V33 was pulsating as an RRd star in 1950, but that during the interval 1986-1991, it had either stopped, or the oscillations in the fundamental mode were at a very low level, too low to be detected by photographic observations. A similar analysis of the observations of V34 in the same data set indicated a primary period 0.4001 with amplitude $3.14(s)$ and $\sigma=0.61$ and a secondary period 0.5367 with amplitude 1.63 and $\sigma=0.61$. The periods and amplitude ratio $\left(A_{1} / A_{0}=1.93\right)$ derived from these earlier observations for V34 are comparable to the results obtained from the Las Campanas data used in the present investigation (see Table 4). This gives support to the hypothesis that the pulsation properties of V33 changed dramatically between 1950 and 1986 . It is also important to note that the period of V33 is longer than the periods of all of the other RRc variables in M68, and shorter than the primary periods of all of the RRd stars, with the exception of V7. One might expect such a star to change from an RRd to an RRc type variable.

TABLE 3

B MAgnitudes For 30 RR LyRAe VARIables in M68

\begin{tabular}{ccccccccccccccccc}
\hline \hline Hel. JD & V1 & V2 & V3 & V4 & V5 & V6 & V7 & V8 & V9 & V11 & V13 & V14 & V15 & V16 & V18 \\
\hline 46552.543 & 16.31 & 15.78 & 16.24 & 15.87 & 16.14 & 16.29 & 16.07 & 15.83 & 15.87 & 15.54 & 15.90 & 16.43 & 15.90 & 15.76 & 16.35 \\
$\ldots$ & $\ldots$ & $\ldots$ & $\ldots$ & $\ldots$ & $\ldots$ & $\ldots$ & $\ldots$ & $\ldots$ & $\ldots$ & $\ldots$ & $\ldots$ & $\ldots$ & $\ldots$ & $\ldots$ & $\ldots$ \\
$\ldots$ & $\ldots$ & $\ldots$ & $\ldots$ & $\ldots$ & $\ldots$ & $\ldots$ & $\ldots$ & $\ldots$ & $\ldots$ & $\ldots$ & $\ldots$ & $\ldots$ & $\ldots$ & $\ldots$ & $\ldots$ \\
48396.589 & 16.28 & 16.39 & 15.86 & 16.07 & 15.75 & 15.77 & 16.25 & 16.30 & 16.07 & 15.69 & 15.52 & 16.61 & 15.56 & 16.16 & 16.30 \\
\hline \hline Hel. JD & V19 & V20 & V21 & V22 & V23 & V24 & V25 & V26 & V28 & V29 & V30 & V31 & V32 & V33 & V34 \\
\hline 46552.543 & 15.94 & 16.03 & 15.16 & 15.56 & 16.20 & 16.14 & 15.72 & 16.37 & 16.29 & 16.22 & 16.16 & 16.01 & 15.94 & 16.35 & 16.07 \\
$\ldots$ & $\ldots$ & $\ldots$ & $\ldots$ & $\ldots$ & $\ldots$ & $\ldots$ & $\ldots$ & $\ldots$ & $\ldots$ & $\ldots$ & $\ldots$ & $\ldots$ & $\ldots$ & $\ldots$ & $\ldots$ \\
$\ldots$ & $\ldots$ & $\ldots$ & $\ldots$ & $\ldots$ & $\ldots$ & $\ldots$ & $\ldots$ & $\ldots$ & $\ldots$ & $\ldots$ & $\ldots$ & $\ldots$ & $\ldots$ & $\ldots$ \\
48396.556 & 15.87 & 15.75 & 15.55 & 16.21 & 15.51 & 16.15 & 16.35 & 15.67 & 16.30 & 15.72 & 16.16 & 15.84 & 16.21 & 15.73 & 15.82 \\
\hline
\end{tabular}

Complete table available in ApJ CD-ROM series. 
TABLE 4

ElEMENTS OF THE RR LyRaE VARIables IN M68 (based on the Las Campanas observations)

\begin{tabular}{|c|c|c|c|c|c|c|}
\hline Star & $\begin{array}{l}\text { Bailey } \\
\text { Type }\end{array}$ & $x^{\prime \prime}$ & $y^{\prime \prime}$ & $\begin{array}{c}\text { Period } \\
\text { (days) }\end{array}$ & $\langle B\rangle$ & $A_{B}$ \\
\hline V1 ...... & c & -283 & 109 & 0.349574 & 15.96 & 0.72 \\
\hline V2 ...... & $a b$ & -168 & -44 & 0.578185 & 16.12 & 1.04 \\
\hline V3 $\ldots . .$. & d & -140 & 91 & 0.390734 & 16.04 & 0.63 \\
\hline V4 ...... & d & -118 & -132 & 0.395540 & 16.00 & 0.52 \\
\hline V5 ...... & c & -53 & 169 & 0.282117 & 15.97 & 0.50 \\
\hline V6. & c & -54 & 17 & 0.368493 & 15.90 & 0.61 \\
\hline V7 ...... & d & -51 & -58 & 0.387958 & 15.99 & 0.53 \\
\hline V8 ...... & $\mathrm{c}$ & -35 & -134 & 0.390407 & 16.00 & 0.57 \\
\hline V9 ...... & $a b$ & -30 & 40 & 0.579045 & 15.75 & 0.81 \\
\hline V11 ..... & $\mathrm{c}$ & -17 & -113 & 0.364931 & 15.87 & 0.65 \\
\hline V13 ..... & $\mathrm{c}$ & -4 & -57 & 0.361742 & 15.81 & 0.72 \\
\hline V14 ..... & $a b$ & -2 & 218 & 0.556803 & 16.07 & 1.40 \\
\hline V15 ..... & c & 10 & 59 & 0.372269 & 15.52 & 0.65 \\
\hline V16 ..... & c & 10 & 78 & 0.381960 & 15.94 & 0.59 \\
\hline V18 ..... & c & 18 & -96 & 0.367348 & 15.99 & 0.64 \\
\hline V19 ..... & d & 32 & 70 & 0.391633 & 15.86 & 0.57 \\
\hline V20 ..... & c & 33 & -114 & 0.385794 & 15.96 & 0.59 \\
\hline V21 .... & $d$ & 46 & 8 & 0.400355 & 15.25 & 0.59 \\
\hline $\mathrm{V} 22 \ldots .$. & $a b$ & 61 & -22 & 0.563439 & 15.72 & 1.41 \\
\hline V23 ..... & $a b$ & 65 & 380 & 0.658884 & 16.02 & 1.22 \\
\hline V24 ..... & $\mathrm{c}$ & 72 & -8 & 0.376473 & 15.90 & 0.59 \\
\hline V25 ..... & $a b$ & 140 & 123 & 0.641490 & 16.04 & 1.00 \\
\hline V26 ..... & d & 157 & -45 & 0.407031 & 16.08 & 0.55 \\
\hline V28 ..... & $a b$ & 439 & 159 & 0.606780 & 16.06 & 1.21 \\
\hline V29 .... & $\mathrm{d}$ & 283 & -153 & 0.395236 & 16.03 & 0.53 \\
\hline V30 ..... & $a b$ & 112 & -77 & 0.733635 & 16.08 & 0.50 \\
\hline V31 .... & d & -109 & 96 & 0.399655 & 15.97 & 0.54 \\
\hline V32 .... & $a b$ & -330 & -639 & 0.588187 & 16.02 & 1.08 \\
\hline V33 ..... & c & 89 & 59 & 0.390569 & 15.94 & 0.62 \\
\hline V34 ..... & $\mathrm{d}$ & 268 & 216 & 0.400134 & 16.03 & 0.53 \\
\hline
\end{tabular}

The results of our period searches are summarized in Table 4 which lists, for each star, the Bailey type, the $x, y$ position (in arcsec) relative to the cluster center according to Sawyer Hogg (1973), the primary period, the (intensity) mean $B$ magnitude, and the $B$ amplitude, derived by fitting equation (1) to the light curve. For the RRab stars, the fit to equation (1) was of order $n=6$, and for the other stars, it was $n=4$. The data of Table 4 indicate that stars which lie closer to the cluster center (V9, V13, V15, V21, and V22) appear brighter than the others. This is due to the effects of crowding. Light curves for the $30 \mathrm{RR}$ Lyrae stars are shown in Figure 1. The curves are arranged in order of increasing period, and apart from the five crowded stars, V9, V13, V15, V21, and V22, it can be seen that stars with periods in the range 0.39 to 0.41 have more scatter than the others on their light curves. this is caused by the presence of double-mode pulsations. (The excessive scatter for V21 is due partly to double-mode pulsations and partly to the effects of crowding. On many of the plates, its magnitude had to be extrapolated from the calibration curve because it was brighter than the standard stars.) For some of the RRab (fundamental mode) stars (V9, V25, and V32), variations in the amplitude occurred during the interval of observations. However, our analysis indicated that these variations were not due to simultaneous pulsations in the first overtone mode, but were probably caused by the Blazhko effect.

\section{THE DOUBLE-MODE RR LYRAE VARIABLES}

The characteristics of the light curves of the double-mode stars are listed in Table 5. The adopted primary and secondary periods were determined by Stobie's (1970) iterative procedure. The amplitudes were determined by fitting equation (1) to the light curves. The standard deviations of the points about these fitted curves are also included in the table. For six of the stars (V3, V4, V7, V26, V31, and V34), the detected fundamental mode pulsations have a high signal-to-noise ratio, i.e., $A_{0} / \sigma_{0}$ is greater than 2. However, for V19 and V29, they are weaker, and this makes it impossible to determine the secondary periods unambiguously. Therefore, the values of $P_{0}$ and $P_{1} / P_{0}$ are uncertain for these two stars. The star V21 is omitted from Table 5 because it was not possible to determine a reliable value of the secondary period. This was partly because of the crowding, but it also appears that the strength of the secondary pulsations varied during the interval of the observations. The analysis of all the observations indicated a primary period of 0.40 with amplitude and standard deviation of the points around the light curve: $A=0.59, \sigma=0.29$ and no significant secondary pulsations. However, when the 1986, 1987, and 1988 observations were analyzed alone, a different result was obtained. For these observations, we found a primary period of approximately 0.40 and a secondary period of approximately 0.54 , with $A_{1}=0.47, \sigma_{1}=0.13$ for the first overtone and $A_{0}=$ $0.50, \sigma_{0}=0.13$ for the fundamental mode. However, the analysis of the 1989, 1990, and 1991 observations gave no evidence for fundamental mode pulsations. It appears that, during the interval 1986-1988, the star V21 was pulsating simultaneously in the fundamental and first overtone modes, and the amplitudes of the two modes were approximately equal. The fact that the first overtone period is longer than 0.4 makes this hypothesis plausible. However, when the star was observed later, only the first overtone oscillations were present. Thus, it seems that double-mode pulsation in V21 is a transient phenomenon. It is unfortunate that the magnitudes determined for this star were so uncertain. Clearly, it is a good candidate for future observations with a CCD detector. In fact, it would be important to continue to monitor the pulsation properties of all of these RRc and RRd stars because we already noted in $\S 3$ that the double-mode properties of V33 have changed as well. The data of Table 5 show that, among the other eight RRd stars, the strongest fundamental mode oscillations

TABLE 5

Characteristics OF THE double-MOde STARS IN M68 (Las Campanas observations)

\begin{tabular}{|c|c|c|c|c|c|c|}
\hline Star & $\begin{array}{c}P_{1} \\
\text { (days) }\end{array}$ & $\begin{array}{c}A_{1} \\
\left(\sigma_{1}\right)\end{array}$ & $\begin{array}{c}P_{0} \\
\text { (days) }\end{array}$ & $\begin{array}{c}A_{0} \\
\left(\sigma_{0}\right)\end{array}$ & $P_{1} / P_{0}$ & $A_{1} / A_{0}$ \\
\hline V3 ...... & 0.390734 & $\begin{array}{c}0.59 \\
(0.08)\end{array}$ & 0.523601 & $\begin{array}{c}0.29 \\
(0.08)\end{array}$ & 0.7462 & 2.03 \\
\hline V4 ...... & 0.395940 & $\begin{array}{c}0.53 \\
(0.07)\end{array}$ & 0.530534 & $\begin{array}{c}0.17 \\
(0.07)\end{array}$ & 0.7463 & 3.12 \\
\hline v7 ...... & 0.387958 & $\begin{array}{c}0.53 \\
(0.07)\end{array}$ & 0.519740 & $\begin{array}{c}0.19 \\
(0.07)\end{array}$ & 0.7464 & 2.79 \\
\hline V19 ..... & 0.391633 & $\begin{array}{c}0.56 \\
(0.10)\end{array}$ & 0.526207 & $\begin{array}{c}0.19 \\
(0.10)\end{array}$ & 0.7443: & 2.95 \\
\hline V26..... & 0.407031 & $\begin{array}{c}0.51 \\
(0.08)\end{array}$ & 0.546246 & $\begin{array}{c}0.35 \\
(0.08)\end{array}$ & 0.7451 & 1.46 \\
\hline V29..... & 0.395236 & $\begin{array}{c}0.53 \\
(0.05)\end{array}$ & 0.530775 & $\begin{array}{c}0.10 \\
(0.05)\end{array}$ & 0.7446 : & 5.30 \\
\hline V31 ..... & 0.399655 & $\begin{array}{c}0.55 \\
(0.08)\end{array}$ & 0.535498 & $\begin{array}{c}0.23 \\
(0.08)\end{array}$ & 0.7463 & 2.20 \\
\hline V34 ..... & 0.400134 & $\begin{array}{c}0.53 \\
(0.08)\end{array}$ & 0.536518 & $\begin{array}{c}0.27 \\
(0.08)\end{array}$ & 0.7458 & 1.96 \\
\hline
\end{tabular}



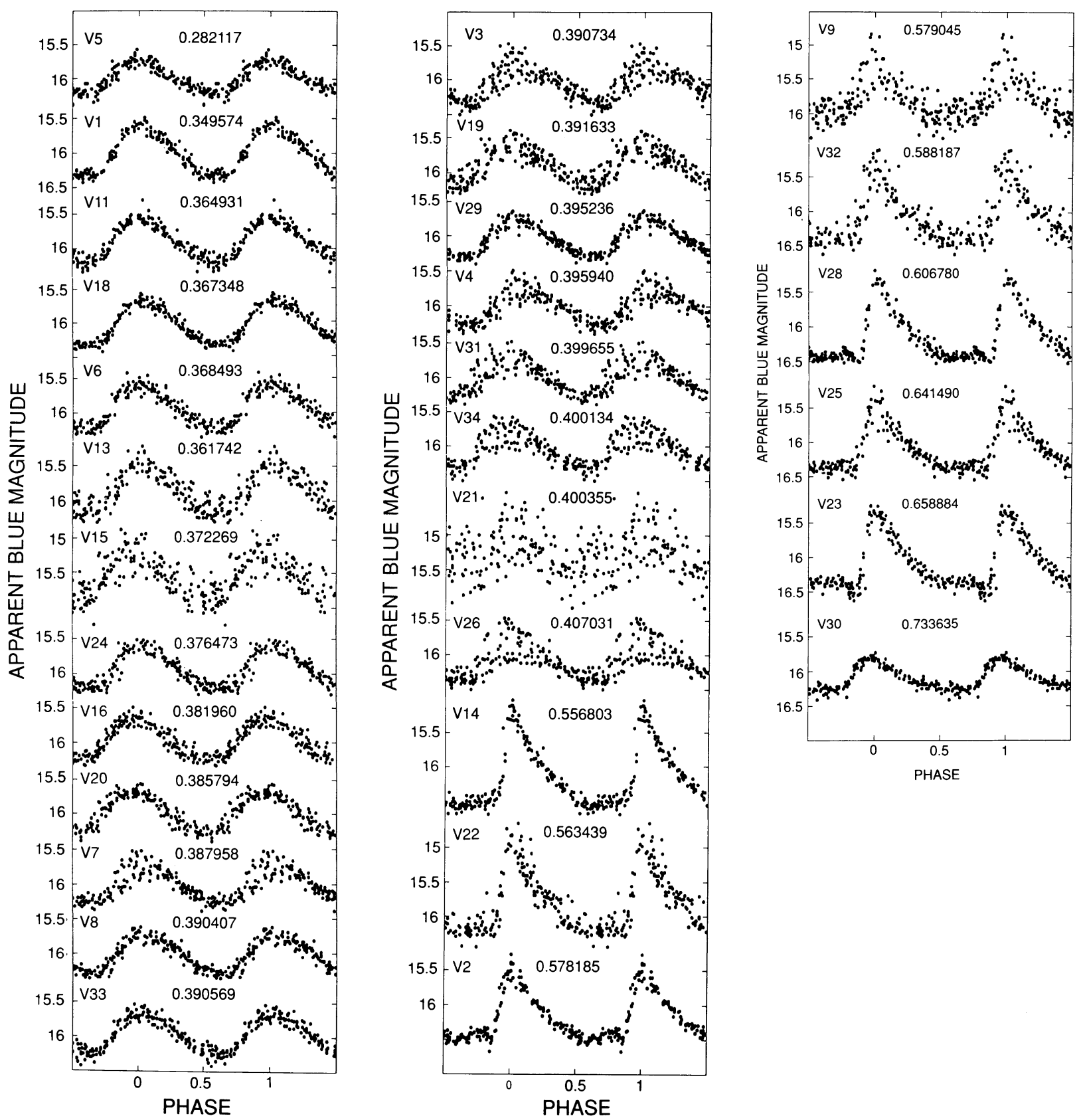

FIG. 1.- Light curves ( $B$ mag) for 30 RR Lyrae variables in M68. The stars are arranged in order of increasing period, and the period is labeled above each curve.

(smallest amplitude ratio $A_{1} / A_{0}$ ) have been detected in V26 and V34, the two stars with the longest periods. Apparently these two stars have recently been (or are about to become) RRab variables.

\section{MASSES OF THE DOUBLE-MODE RR LYRAE VARIABLES}

The first mass determinations for RRd stars in globular clusters were by Cox et al. (1983). From models computed with Los Alamos opacities, they found that the mean mass for seven RRd stars in the metal-poor Oosterhoff type II cluster, M15, was $0.65 M_{\odot}$, compared with $0.55 M_{\odot}$ for two RRd stars in the Oosterhoff type I cluster, M3, which has a moderate metal abundance. At the time, it was generally assumed that the mass of an RRd star could be computed from its period, period ratio and helium abundance and that the metal abundance was not an important parameter. This assumption is no longer considered valid. Models based on new OPAL opacities (Rogers \& Iglesias 1992) indicate that the metallicity is very important. Consequently, recent investigations of Cox (1991), Kovács, Buchler, \& Marom (1991), and Kovács et al. (1992), based on the OPAL opacities, all suggest that the masses of the RRd stars are greater than previously thought, and that those for the RRd stars in Osterhoff type I clusters could possibly be as large as those in the Oosterhoff type II clusters.

A Petersen diagram $\left(\boldsymbol{P}_{1} / \boldsymbol{P}_{0}\right.$ vs. $\left.\boldsymbol{P}_{0}\right)$ is shown in Figure 2 . The points for M68 are represented by open triangles, but the two stars with uncertain period ratios (V19 and V29) are not plotted. Also included in the diagram are the double-mode variables in other galactic globular clusters. The periods and period ratios for these stars and the metallicities of the clusters 


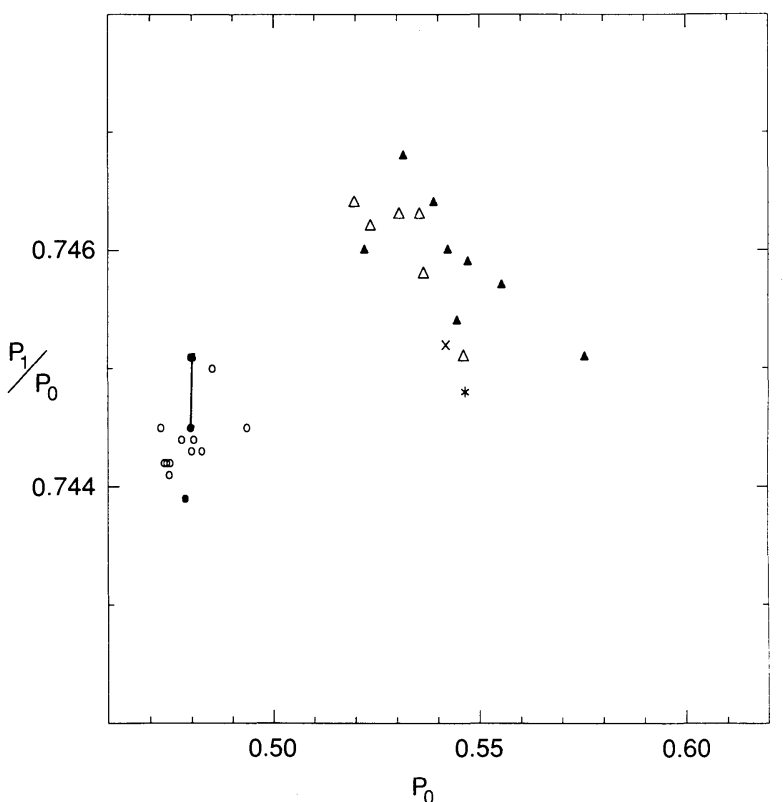

Fig. 2.-Petersen (1973) $P_{1} / P_{0}$ vs. $P_{0}$ diagram. The galactic globular cluster RRd stars are plotted: open triangles (M68), solid triangles (M15), asterisk (NGC 2419), cross (NGC 6426), open circles (IC 4499), and solid circles (M3).

to which they belong are listed in Table 6, and come from the following sources: IC 4499 (Clement et al. 1986; Clement \& Walker 1990), M3 (Nemec \& Clement 1989), NGC 2419, and NGC 6426 (Clement \& Nemec 1990). The periods and period ratios for M15 are from Clement \& Walker (1990) and are their mean values determined from published magnitudes for observations made in the years 1954-1959 and 1976-1979. There are other RRd stars in M15, but their secondary periods could not be established unambiguously. The metal abundances given in Table 6 are those published by Suntzeff et al. (1991). Also
TABLE 6

Periods and Period Ratios of Double-Mode RR Lyrae Stars in Galactic Globular Clusters and in the Field

\begin{tabular}{|c|c|c|c|c|}
\hline Cluster & {$[\mathrm{Fe} / \mathrm{H}]$} & Star & $P_{0}$ & $P_{1} / P_{0}$ \\
\hline \multirow[t]{11}{*}{ IC $4499 \ldots \ldots \ldots$} & -1.50 & V8 & 0.4935 & 0.7445 \\
\hline & & V10 & 0.4746 & 0.7441 \\
\hline & & V18 & 0.4734 & 0.7442 \\
\hline & & V21 & 0.4748 & 0.7442 \\
\hline & & V42 & 0.4852 & 0.7450 \\
\hline & & V59 & 0.4800 & 0.7443 \\
\hline & & V65 & 0.4740 & 0.7442 \\
\hline & & V73 & 0.4825 & 0.7443 \\
\hline & & V78 & 0.4726 & 0.7445 \\
\hline & & V87 & 0.4805 & 0.7444 \\
\hline & & V109 & 0.4776 & 0.7444 \\
\hline \multirow[t]{2}{*}{ M3 $\ldots \ldots \ldots \ldots$} & -1.66 & V68 & 0.4785 & 0.7439 \\
\hline & & V87 & 0.4798 & $\begin{array}{l}0.7451: \\
0.7445:\end{array}$ \\
\hline NGC $2419 \ldots .$. & -2.10 & V39 & 0.5465 & 0.7448 \\
\hline \multirow[t]{8}{*}{ M15 ............ } & -2.17 & V17 & 0.5756 & 0.7451 \\
\hline & & V26 & 0.5390 & 0.7464 \\
\hline & & V30 & 0.5446 & 0.7454 \\
\hline & & V31 & 0.5472 & 0.7459 \\
\hline & & V39 & 0.5222 & 0.7460 \\
\hline & & V51 & 0.5316 & 0.7468 \\
\hline & & V53 & 0.5554 & 0.7457 \\
\hline & & V67 & 0.5424 & 0.7460 \\
\hline NGC $6426 \ldots .$. & -2.20 & V3 & 0.5419 & 0.7452 \\
\hline \multirow[t]{3}{*}{ Field $\ldots . \ldots \ldots \ldots$} & -1.81 & AQ Leo & 0.5498 & 0.7461 \\
\hline & -1.86 & VIII-10 & 0.5118 & 0.7449 \\
\hline & -1.77 & VIII-58 & 0.4647 & 0.7432 \\
\hline
\end{tabular}

included in Table 6 is the same information for three RRd stars in the field. The data for these stars are from Jerzykiewcz, Schult, \& Wenzel (1982), Mendes de Oliveira \& Smith (1990), and Clement, Kinman, \& Suntzeff(1991).

Table 7 shows the masses of individual RRd stars in M68 and M15, derived as a function of luminosity, according to the fitting formulas given by Kovács et al. (1991). The metallicity was taken to be $Z=0.0001$. The masses and $\log L$ are in solar

TABLE 7

MASSES FOR RRd VARIABLES IN M68 AND M15

\begin{tabular}{|c|c|c|c|c|c|c|c|c|}
\hline \multirow[b]{2}{*}{ Cluster } & \multirow[b]{2}{*}{ STAR } & \multirow[b]{2}{*}{$\log P_{1}$} & \multicolumn{6}{|c|}{ Mass as a Function of LOG $L$} \\
\hline & & & $\log L=1.68$ & $\log L=1.72$ & $\log L=1.76$ & $\log L=1.80$ & $\log L=1.84$ & $\log L=1.88$ \\
\hline \multirow[t]{6}{*}{ M68................. } & V3 & -0.4081 & 0.75 & 0.75 & 0.77 & 0.79 & 0.82 & 0.85 \\
\hline & V4 & -0.4024 & 0.76 & 0.76 & 0.78 & 0.80 & 0.82 & 0.86 \\
\hline & V7 & -0.4112 & 0.75 & 0.76 & 0.77 & 0.79 & 0.82 & 0.86 \\
\hline & V26 & -0.3904 & 0.73 & 0.73 & 0.75 & 0.76 & 0.79 & 0.83 \\
\hline & V31 & -0.3983 & 0.77 & 0.77 & 0.78 & 0.80 & 0.83 & 0.87 \\
\hline & V34 & -0.3978 & 0.75 & 0.75 & 0.76 & 0.78 & 0.81 & 0.85 \\
\hline \multirow[t]{2}{*}{ M68 .................. } & \multirow{2}{*}{\multicolumn{2}{|c|}{$\begin{array}{l}\text { Mean mass } \\
\text { Mean } T_{e}\end{array}$}} & 0.75 & 0.75 & 0.77 & 0.79 & 0.82 & 0.85 \\
\hline & & & 6489 & 6634 & 6753 & 6872 & 6979 & 7089 \\
\hline \multirow[t]{8}{*}{ M15 .................. } & V17 & -0.3676 & 0.77 & 0.77 & 0.77 & 0.79 & 0.82 & 0.86 \\
\hline & V26 & -0.3955 & 0.77 & 0.78 & 0.79 & 0.81 & 0.84 & 0.88 \\
\hline & V30 & -0.3915 & 0.74 & 0.75 & 0.76 & 0.77 & 0.80 & 0.84 \\
\hline & V31 & -0.3892 & 0.77 & 0.77 & 0.78 & 0.80 & 0.82 & 0.86 \\
\hline & V39 & -0.4094 & 0.74 & 0.74 & 0.76 & 0.78 & 0.81 & 0.85 \\
\hline & V51 & -0.4013 & 0.78 & 0.79 & 0.80 & 0.82 & 0.84 & 0.88 \\
\hline & V53 & -0.3829 & 0.77 & 0.77 & 0.78 & 0.80 & 0.82 & 0.86 \\
\hline & V67 & -0.3930 & 0.76 & 0.77 & 0.78 & 0.80 & 0.82 & 0.86 \\
\hline \multirow[t]{2}{*}{ M15 ................. } & \multirow{2}{*}{\multicolumn{2}{|c|}{$\begin{array}{l}\text { Mean mass } \\
\text { Mean } T_{e}\end{array}$}} & 0.76 & 0.77 & 0.78 & 0.80 & 0.82 & 0.86 \\
\hline & & & 6428 & 6557 & 6690 & 6808 & 6929 & 7024 \\
\hline
\end{tabular}

Masses are based on models of Kovacs, Buchler, \& Marom 1991 for $Z=0.0001$. 
units. For each cluster, the last two rows in Table 7 give the mean mass and temperature (the latter calculated from the period/mean density law) of the RRd sample, again as a function of luminosity. One sees that the derived mean RRd mass increases by about $0.1 M_{\odot}$ as the assumed mean luminosity is raised by 0.2 in $\log L$. For comparison, further calculations show that the effect of increasing $Z$ to 0.0002 is to raise the inferred RRd masses by about $0.05 M_{\odot}$. This latter is a relatively modest effect, unlike the case for OoI clusters, where changing the metallicity by a factor of 2 would alter the RRd masses drastically (Kovács et al. 1991).

Taken together, Table 7 and Figure 2 demonstrate the great similarity of the RRd samples in M68 and M15. In the next section, we shall see that the RRc stars in these two clusters are also very similar.

\section{FOURIER DECOMPOSITION OF THE LIGHT CURVES}

In a recent study, Clement, Jankulak, \& Simon (1992; hereafter CJS) analyzed five globular clusters and demonstrated that the RRc stars in a given cluster show a sequence in which the Fourier phase parameter, $\phi_{31}$, increases with period, and that the higher the metallicity, the higher the sequence lies in a plot of $\phi_{31}$ vesus period. To establish the place of the M68 variables in this sequence, we fitted equation (1) to the observations and calculated the low-order Fourier coefficients:

$$
R_{j 1}=A_{j} / A_{1}, \quad \phi_{j 1}=\phi_{j}-j \phi_{1}, \quad j=1,2,3,4 .
$$

For each star, the period used was the value listed in Table 4 and the epoch was taken as JD $2,400,000.000$ so that $t$ in equation (1) refers to (JD - 2,400,000.000) where JD represents the Julian date of the observation. For the double-mode variables, Fourier parameters were determined for the first overtone mode, although it is not known precisely how the Fourier coefficients of the dominant mode should compare with those for the RRc stars. The magnitudes for the RRd stars were fitted to the relation:

$$
\operatorname{mag}=A_{0}+\sum A_{j}^{i} \cos \left[\left(i \omega_{0}+j \omega_{1}\right) t+\phi_{j}^{i}\right],
$$

where the indices $i$ and $j$ run over all positive and negative integers, including zero, subject to the restrictions:

$$
0<|i|+|j| \leq n, \quad i+j \geq 0, \quad j>0, \quad \text { when } i+j=0
$$

(Simon 1979). In equation (2), $\omega_{0}$ refers to the fundamental mode and $\omega_{1}$ refers to the first overtone. The periods $P_{0}$ and $P_{1}$ used in the calculations were those listed in Table 5. For these RRd stars, the order of the fit was $n=3, \phi_{j 1}$ for the overtone mode was computed from $\phi_{j}^{0}-j \phi_{1}^{0}$, and $R_{j 1}$ as $A_{j}^{0} / A_{1}^{0}$. Following CJS, we used Efron's bootstrap procedure (Diaconis \& Efron 1983) to assess the uncertainties in the calculated values of the Fourier parameters. The Fourier parameters and their errors $\left(\sigma_{\text {Boot }}\right)$ for the RRc and RRd variables are listed in Table 8A. Although, for this investigation, we are interested only in the properties of these stars, we include our results for the RRab stars as well, in Table 8B. The parameters listed in Tables $8 \mathrm{~A}$ and $8 \mathrm{~B}$ are the number of observations, period, amplitude, standard deviation of the fit $(\sigma)$, the Fourier coefficients $A_{0}, A_{1}$, the amplitude ratios $R_{21}$ to $R_{41}, \phi_{1}$, and the phase differences $\phi_{21}$ to $\phi_{41}$. The stars are arranged in order of increasing period so that any systematic changes of the parameters with period can be readily noted. In general, the values of the amplitude ratios and phase differences for the variables in
M68 fall within the ranges for these parameters for RR Lyrae stars in the field (Simon \& Teays 1982) and in $\omega$ Centauri (Petersen 1984).

Figure 3 shows a plot of $\phi_{31}$ versus $\log P$ for the stars in M68 (plotted as solid stars) with the most reliable values of $\phi_{31}$. Based on artificial data simulation, CJS found that when the true errors in $\phi_{31}$ are greater than 0.5 , the bootstrap method underestimates the errors, but when the true errors are smaller, the method overestimates them. Because of this result, they included, in their study, only stars for which $\sigma_{\text {Boot }}$ was less than 0.4. According to Table $8 \mathrm{~A}$, there are seven stars that satisfy this criterion. These are the RRc stars, V1, V8, V13, V18, V24, and V33, and the RRd star, V3. Although it appears from the light curves of Figure 1 and the $\sigma$ values in column (5) of Table 8A that the observations for V13, which has $\sigma_{\mathrm{Boot}}=0.36$, may not be as good as for the other stars (due to crowding effects), we have included it anyway for consistency with the earlier study.

Also included in Figure 3, for comparison, are the $\phi_{31}$ values determined by CJS for the Las Campanas observations of the RRc stars in the more metal rich clusters, NGC 6171 (open circles) and M5 (solid circles), and in the metal-poor cluster M15 (open triangles). It is clear that the M68 points show the characteristic rise of $\phi_{31}$ with period, and that they fall so closely among the M15 stars as to make the two extreme metal-poor clusters virtually indistinguishable on the diagram. Thus the RRc samples are seen to confirm the similarity of M68 and M15 already noted in the case of the RRd stars. It is unfortunate that reliable Fourier parameters are available for so few of the large sample of RRc and RRd stars in M15 and M68. CCD observations should be made for both clusters, so that the light curves will have less scatter and the parameters can be calculated with greater precision.

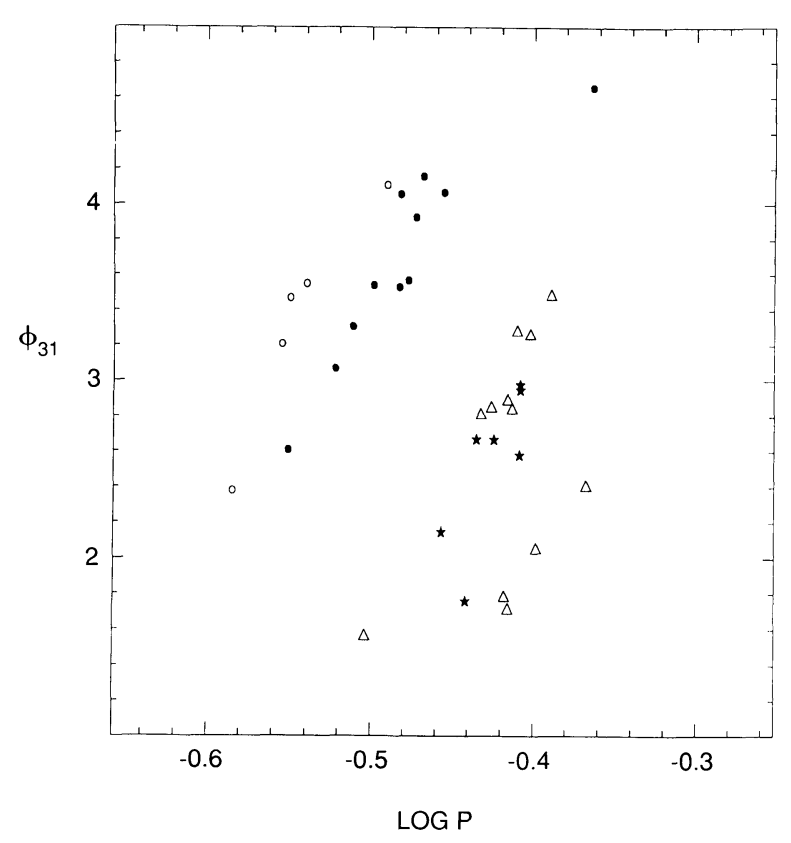

FIG. 3. $-\phi_{31}$ vs. $\log P$ plot for RRc stars in NGC 6171 (open circles), M5 (solid circles), M15 (open triangles), and M68 (solid stars). The metallicities, $[\mathrm{Fe} / \mathrm{H}]$, of these three clusters are $-0.99,-1.40,-2.17$, and -2.09 , for NGC 6171, M5, M15, and M68, respectively. The stars plotted all have errors $\left(\sigma_{\text {Boot }}\right)$ in $\phi_{31}$ of less than 0.40 . 
TABLE 8A

Fourier Parameters for the RRc AND RRd VARIABLES

\begin{tabular}{|c|c|c|c|c|c|c|c|c|c|c|c|c|c|}
\hline \multirow{2}{*}{$\begin{array}{l}\text { Star } \\
\text { (1) }\end{array}$} & \multirow{2}{*}{$\begin{array}{l}\mathrm{N} \\
(2)\end{array}$} & \multirow{2}{*}{$\begin{array}{c}P \text { (days) } \\
\text { (3) }\end{array}$} & \multirow{2}{*}{$\begin{array}{r}A_{B} \\
(4)\end{array}$} & \multirow{2}{*}{$\begin{array}{c}\sigma \\
(5)\end{array}$} & \multirow{2}{*}{$\begin{array}{l}A_{0} \\
(6)\end{array}$} & \multirow{2}{*}{$\begin{array}{l}A_{1} \\
(7)\end{array}$} & \multirow{2}{*}{$\begin{array}{r}R_{21} \\
-(8)\end{array}$} & \multirow{2}{*}{$\begin{array}{c}R_{31} \\
\sigma_{B o o t} \\
(9)\end{array}$} & \multirow{2}{*}{$\begin{array}{l}R_{41} \\
-\quad \\
(10)\end{array}$} & \multirow{2}{*}{$\begin{array}{c}\phi_{1} \\
(11)\end{array}$} & \multirow{2}{*}{$\begin{array}{r}\phi_{21} \\
(12)\end{array}$} & \multirow{2}{*}{$\begin{array}{l}\phi_{31} \\
\sigma_{B o o t} \\
(13)\end{array}$} & \multirow{2}{*}{$\begin{array}{l}\phi_{41} \\
- \\
(14)\end{array}$} \\
\hline & & & & & & & & & & & & & \\
\hline V5 (RRc) & 157 & 0.282117 & 0.500 & 0.068 & 15.986 & 0.239 & $\begin{array}{l}0.217 \\
0.034\end{array}$ & $\begin{array}{l}0.016 \\
0.022\end{array}$ & $\begin{array}{l}0.042 \\
0.027\end{array}$ & 2.94 & $\begin{array}{l}4.24 \\
0.15\end{array}$ & $\begin{array}{l}0.52 \\
1.55\end{array}$ & $\begin{array}{l}1.81 \\
0.79\end{array}$ \\
\hline V1 & 157 & 0.349574 & 0.714 & 0.061 & 15.988 & 0.361 & $\begin{array}{l}0.159 \\
0.020\end{array}$ & $\begin{array}{l}0.085 \\
0.016\end{array}$ & $\begin{array}{l}0.036 \\
0.017\end{array}$ & 3.92 & $\begin{array}{l}4.47 \\
0.15\end{array}$ & $\begin{array}{l}2.15 \\
0.22\end{array}$ & $\begin{array}{l}1.96 \\
0.48\end{array}$ \\
\hline V13 & 157 & 0.361742 & 0.724 & 0.120 & 15.838 & 0.343 & $\begin{array}{l}0.182 \\
0.040\end{array}$ & $\begin{array}{l}0.110 \\
0.033\end{array}$ & $\begin{array}{l}0.029 \\
0.033\end{array}$ & 5.05 & $\begin{array}{l}4.17 \\
0.27\end{array}$ & $\begin{array}{l}1.76 \\
0.36\end{array}$ & $\begin{array}{l}4.34 \\
1.32\end{array}$ \\
\hline V11 & 157 & 0.364931 & 0.646 & 0.067 & 15.889 & 0.317 & $\begin{array}{l}0.180 \\
0.023\end{array}$ & $\begin{array}{l}0.027 \\
0.020\end{array}$ & $\begin{array}{l}0.018 \\
0.017\end{array}$ & 0.79 & $\begin{array}{l}4.37 \\
0.13\end{array}$ & $\begin{array}{l}2.74 \\
0.96\end{array}$ & $\begin{array}{l}1.61 \\
1.08\end{array}$ \\
\hline V18 & 157 & 0.367348 & 0.634 & 0.054 & 16.020 & 0.326 & $\begin{array}{l}0.145 \\
0.020\end{array}$ & $\begin{array}{l}0.081 \\
0.018\end{array}$ & $\begin{array}{l}0.023 \\
0.015\end{array}$ & 1.27 & $\begin{array}{l}4.49 \\
0.14\end{array}$ & $\begin{array}{l}2.67 \\
0.23\end{array}$ & $\begin{array}{l}1.46 \\
0.68\end{array}$ \\
\hline V6 & 157 & 0.368493 & 0.615 & 0.076 & 15.921 & 0.319 & $\begin{array}{l}0.167 \\
0.028\end{array}$ & $\begin{array}{l}0.079 \\
0.028\end{array}$ & $\begin{array}{l}0.061 \\
0.022\end{array}$ & 4.75 & $\begin{array}{l}4.56 \\
0.20\end{array}$ & $\begin{array}{l}3.15 \\
0.44\end{array}$ & $\begin{array}{l}1.79 \\
0.42\end{array}$ \\
\hline V24 & 157 & 0.376473 & 0.592 & 0.074 & 15.928 & 0.309 & $\begin{array}{l}0.164 \\
0.023\end{array}$ & $\begin{array}{l}0.087 \\
0.021\end{array}$ & $\begin{array}{l}0.054 \\
0.025\end{array}$ & 2.74 & $\begin{array}{l}4.22 \\
0.13\end{array}$ & $\begin{array}{l}2.67 \\
0.30\end{array}$ & $\begin{array}{l}1.53 \\
0.46\end{array}$ \\
\hline V16 & 157 & 0.381960 & 0.586 & 0.077 & 15.959 & 0.295 & $\begin{array}{l}0.140 \\
0.026\end{array}$ & $\begin{array}{l}0.060 \\
0.028\end{array}$ & $\begin{array}{l}0.039 \\
0.021\end{array}$ & 1.73 & $\begin{array}{l}4.11 \\
0.21\end{array}$ & $\begin{array}{l}3.31 \\
0.59\end{array}$ & $\begin{array}{l}2.46 \\
0.65\end{array}$ \\
\hline V20 & 157 & 0.385794 & 0.593 & 0.078 & 15.979 & 0.303 & $\begin{array}{l}0.092 \\
0.023\end{array}$ & $\begin{array}{l}0.069 \\
0.026\end{array}$ & $\begin{array}{l}0.012 \\
0.021\end{array}$ & 5.53 & $\begin{array}{l}4.47 \\
0.25\end{array}$ & $\begin{array}{l}3.93 \\
0.47\end{array}$ & $\begin{array}{l}1.33 \\
1.81\end{array}$ \\
\hline V8 & 157 & 0.390407 & 0.573 & 0.066 & 16.016 & 0.284 & $\begin{array}{l}0.155 \\
0.028\end{array}$ & $\begin{array}{l}0.105 \\
0.020\end{array}$ & $\begin{array}{l}0.011 \\
0.018\end{array}$ & 3.81 & $\begin{array}{l}4.68 \\
0.20\end{array}$ & $\begin{array}{l}2.58 \\
0.26\end{array}$ & $\begin{array}{l}3.97 \\
1.45\end{array}$ \\
\hline V33 & 157 & 0.390569 & 0.591 & 0.071 & 15.961 & 0.297 & $\begin{array}{l}0.155 \\
0.029 \\
\end{array}$ & $\begin{array}{l}0.087 \\
0.027 \\
\end{array}$ & $\begin{array}{l}0.026 \\
0.019 \\
\end{array}$ & 2.74 & $\begin{array}{l}4.69 \\
0.16\end{array}$ & $\begin{array}{l}2.98 \\
0.31\end{array}$ & $\begin{array}{l}2.67 \\
0.89\end{array}$ \\
\hline V7 (RRd) & 157 & 0.387958 & 0.669 & 0.064 & 15.995 & 0.263 & $\begin{array}{l}0.180 \\
0.030\end{array}$ & $\begin{array}{l}0.049 \\
0.034\end{array}$ & & 0.47 & $\begin{array}{l}4.55 \\
0.19\end{array}$ & $\begin{array}{l}2.15 \\
0.56\end{array}$ & \\
\hline V3 & 157 & 0.390734 & 0.777 & 0.072 & 16.063 & 0.261 & $\begin{array}{l}0.220 \\
0.036\end{array}$ & $\begin{array}{l}0.081 \\
0.034\end{array}$ & & 4.16 & $\begin{array}{l}4.74 \\
0.18\end{array}$ & $\begin{array}{l}2.95 \\
0.33\end{array}$ & \\
\hline V19 & 157 & 0.391633 & 0.675 & 0.097 & 15.869 & 0.274 & $\begin{array}{l}0.154 \\
0.059\end{array}$ & $\begin{array}{l}0.022 \\
0.034\end{array}$ & & 0.00 & $\begin{array}{l}5.00 \\
0.51\end{array}$ & $\begin{array}{l}3.00 \\
1.53\end{array}$ & \\
\hline V29 & 157 & 0.395236 & 0.431 & 0.058 & 16.047 & 0.272 & $\begin{array}{l}0.177 \\
0.028\end{array}$ & $\begin{array}{l}0.064 \\
0.027\end{array}$ & & 4.41 & $\begin{array}{l}4.50 \\
0.13\end{array}$ & $\begin{array}{l}3.17 \\
0.42\end{array}$ & \\
\hline V4 & 157 & 0.395940 & 0.438 & 0.059 & 16.010 & 0.266 & $\begin{array}{l}0.198 \\
0.035\end{array}$ & $\begin{array}{l}0.037 \\
0.024\end{array}$ & & 4.21 & $\begin{array}{l}4.87 \\
0.15\end{array}$ & $\begin{array}{l}2.57 \\
0.67\end{array}$ & \\
\hline V31 & 157 & 0.399655 & 0.799 & 0.061 & 15.973 & 0.282 & $\begin{array}{l}0.185 \\
0.024\end{array}$ & $\begin{array}{l}0.021 \\
0.022\end{array}$ & & 5.39 & $\begin{array}{l}4.92 \\
0.17\end{array}$ & $\begin{array}{l}3.35 \\
1.06\end{array}$ & \\
\hline V34 & 157 & 0.400134 & 0.678 & 0.069 & 16.045 & 0.258 & $\begin{array}{l}0.174 \\
0.042\end{array}$ & $\begin{array}{l}0.105 \\
0.039\end{array}$ & & 2.70 & $\begin{array}{l}4.86 \\
0.18\end{array}$ & $\begin{array}{l}3.48 \\
0.42\end{array}$ & \\
\hline V26 & 157 & 0.407031 & 0.882 & 0.069 & 16.092 & 0.243 & $\begin{array}{l}0.182 \\
0.032\end{array}$ & $\begin{array}{l}0.082 \\
0.039\end{array}$ & & 0.69 & $\begin{array}{l}5.00 \\
0.18\end{array}$ & $\begin{array}{l}2.90 \\
0.65\end{array}$ & \\
\hline
\end{tabular}

Order $n=4$ for the RRc stars and $n=3$ for the RRd stars.

\section{PERIOD CHANGES OF THE RR LYRAE VARIABLES}

The time interval over which observations of the RR Lyrae variables in M68 are available is approximately $40 \mathrm{yr}$. The cluster was intensively observed during the years 1950-1953: with the Radcliffe reflector at Pretoria, South Africa, in 1950 (van Agt \& Oosterhoff 1959) and at Loiano Observatory in Italy in 1951-1953 (Rosino \& Pietra 1954), and then during the years 1986-1991 for the present investigation. (All of these observations were photographic.) It is expected that an interval of only $40 \mathrm{yr}$ is too short to detect changes caused by evolutionary effects, but it is worthwhile to investigate any changes that may have occurred between 1953 and 1986 because many RR Lyrae variables show period changes on short time scales.

Since we had only two sets of observations, our approach was to determine the period for each data set and then calculate the difference. This technique is feasible in this particular case because of the timing of the observations. At both epochs, observations were made for more than 6 consecutive hours on some nights, over several nights in some months, and in different seasons of the year. This minimized the problem of detecting alias periods. As a result, from the 1950-53 observations, periods could be determined with a precision of approximately $4 \times 10^{-6}$ when $P=0.3$ and $16 \times 10^{-6}$ when $P=0.6$. The 
TABLE 8B

Fourier Parameters for tHe RRab Variables

\begin{tabular}{|c|c|c|c|c|c|c|c|c|c|c|c|c|c|}
\hline \multirow[b]{2}{*}{$\begin{array}{l}\text { Star } \\
(1)\end{array}$} & \multirow[b]{2}{*}{$\begin{array}{l}N \\
(2)\end{array}$} & \multirow[b]{2}{*}{$\begin{array}{c}P \text { (days) } \\
\text { (3) }\end{array}$} & \multirow[b]{2}{*}{$\begin{array}{l}A_{B} \\
(4)\end{array}$} & \multirow[b]{2}{*}{$\begin{array}{c}\sigma \\
(5)\end{array}$} & \multirow[b]{2}{*}{$\begin{array}{l}A_{0} \\
(6)\end{array}$} & \multirow[b]{2}{*}{$\begin{array}{l}A_{1} \\
\text { (7) }\end{array}$} & \multicolumn{3}{|c|}{$\sigma_{\text {Boot }}$} & \multirow[b]{2}{*}{$\begin{array}{c}\phi_{1} \\
(11)\end{array}$} & \multicolumn{3}{|c|}{$\sigma_{\text {Boot }}$} \\
\hline & & & & & & & $\begin{array}{l}R_{21} \\
(8)\end{array}$ & $\begin{array}{l}R_{31} \\
\text { (9) }\end{array}$ & $\begin{array}{l}R_{41} \\
(10)\end{array}$ & & $\begin{array}{l}\phi_{21} \\
(12)\end{array}$ & $\begin{array}{l}\phi_{31} \\
(13)\end{array}$ & $\begin{array}{l}\phi_{41} \\
(14)\end{array}$ \\
\hline \multirow[t]{2}{*}{ V14 .......... } & 157 & 0.556803 & 1.388 & 0.093 & 16.158 & 0.527 & 0.387 & 0.290 & 0.179 & 1.89 & 3.82 & 1.48 & 5.58 \\
\hline & & & & & & & 0.019 & 0.018 & 0.0 & & 0.0 & 0.08 & 0.13 \\
\hline \multirow[t]{2}{*}{ V22 .......... } & 156 & 0.563439 & 1.395 & 0.153 & 15.804 & 0.511 & 0.422 & 0.294 & 0.198 & 1.07 & 3.89 & 1.53 & 5.40 \\
\hline & & & & & & & 0.033 & 0.030 & 0.028 & & 0.09 & 0.14 & 0.26 \\
\hline \multirow[t]{2}{*}{ V2 $\ldots \ldots \ldots \ldots$} & 156 & 0.578185 & 1.028 & 0.070 & 16.171 & 0.407 & 0.380 & 0.262 & 0.195 & 3.49 & 3.74 & 1.27 & 5.63 \\
\hline & & & & & & & 0.017 & 0.016 & 0.016 & & 0.05 & 0.07 & 0.13 \\
\hline \multirow[t]{2}{*}{ V9 ............... } & 157 & 0.579045 & 0.798 & 0.179 & 15.780 & 0.320 & 0.311 & 0.235 & 0.070 & 5.60 & 3.2 & 1.18 & 4.51 \\
\hline & & & & & & & 0.050 & 0.064 & 0.047 & & 0.23 & 0.27 & 0.94 \\
\hline \multirow[t]{2}{*}{ V32 ......... } & 150 & 0.588187 & 1.071 & 0.166 & 16.073 & 0.416 & 0.412 & 0.256 & 0.130 & 5.63 & 3.78 & 1.26 & 5.42 \\
\hline & & & & & & & 0.041 & 0.035 & 0.036 & & 0.11 & 0.20 & 0.39 \\
\hline \multirow[t]{2}{*}{$\mathrm{V} 28 \ldots \ldots \ldots$} & 156 & 0.606780 & 1.220 & 0.068 & 16.128 & 0.437 & 0.442 & 0.326 & 0.216 & 3.34 & 3.70 & 1.38 & 5.51 \\
\hline & & & & & & & 0.018 & 0.021 & 0.027 & & 0.05 & 0.06 & 0.12 \\
\hline \multirow[t]{2}{*}{$\mathrm{V} 25 \ldots \ldots \ldots$} & 157 & 0.641490 & 0.981 & 0.096 & 16.089 & 0.388 & 0.411 & 0.279 & 0.156 & 0.69 & 3.75 & 1.52 & 5.58 \\
\hline & & & & & & & 0.022 & 0.034 & 0.029 & & 0.06 & 0.11 & 0.23 \\
\hline \multirow[t]{2}{*}{ V23 .......... } & 157 & 0.658884 & 1.238 & 0.095 & 16.087 & 0.426 & 0.496 & 0.331 & 0.209 & 1.34 & 3.69 & 1.61 & 5.84 \\
\hline & & & & & & & 0.020 & 0.022 & 0.021 & & 0.05 & 0.09 & 0.11 \\
\hline \multirow[t]{2}{*}{$\mathrm{V} 30 \ldots \ldots \ldots$} & 157 & 0.733635 & 0.467 & 0.050 & 16.095 & 0.217 & 0.389 & 0.145 & 0.087 & 0.63 & 3.98 & 2.00 & 6.28 \\
\hline & & & & & & & 0.024 & 0.030 & 0.028 & & 0.08 & 0.16 & 0.29 \\
\hline
\end{tabular}

Order $n=6$ for the RRab stars.

corresponding figures for the 1986-1991 observations are $2.5 \times 10^{-6}$ when $P=0.3$ and $10 \times 10^{-6}$ when $P=0$.6. In order to combine the Loiano observations with the Radcliffe ones, we had to apply a correction to the latter because van Agt \& Oosterhoff's published observations were not on a standard system. They made visual estimates (s) on a scale of $0-6$, and, in addition, they measured 30 of the variables with a Schilt photometer to derive provisional magnitudes. The zero point and scale of these magnitudes were different for different stars. The scaling procedure devised by Clement (1990) was used to transform the Radcliffe provisional magnitudes to the system of Rosino \& Pietra, so that the two data sets could be analyzed together. For four of the stars (V14, V23, V25, and V28), the phase coverage for the Radcliffe data was not adequate for determining the magnitude at maximum light, making it impossible to calculate the correct scaling factor. For these stars, only the Loiano observations were used. Also, there were a number of stars that were observed at the Radcliffe Observatory, but not at Loiano. These stars were not included in the analysis because their periods at the earlier epoch could not be determined with the required precision. In all, 23 stars were studied for period changes. Our results are summarized in Table 9. The periods at both epochs are listed, and for the RRd stars, the periods listed are the primary periods. The values for $\Delta P$ are the differences, $P(1990)-P(1950)$, and the errors depend both on the length of the period and on the amount of scatter on the individual light curves. The value of $\alpha$, the period change rate measured in units of cycles per million years has been calculated on the assumption that $\Delta P$ represents the change of period in the $41 \mathrm{yr}$ interval and that the period changes linearly with time. Typical errors for $\alpha$ are 0.45 for the $\mathrm{RRc}, \mathrm{d}$ stars and greater than 0.6 for the RRab stars. Therefore, most of the errors are larger than the actual period changes detected. For 10 of the stars, the derived period changes were larger than the quoted errors, but only two (V1 and V20) showed changes that were significant at the $2 \sigma$ level.

It is interesting to compare these period changes with those determined for the RR Lyrae variables in M15 by Smith \&
Sandage (1981) because M15 and M68 are similar in a number of respects, including, as shown above, their samples of RRc and RRd stars. According to McClure et al. (1987), their colormagnitude diagrams are similar as well. Smith \& Sandage's study of M15 was based on observations made over an interval of approximately $80 \mathrm{yr}$. Since observations were available from 13 different epochs, they used Belserene's (1964) method for their analysis. This involved plotting an $O-C$ diagram, i.e.,

TABLE 9

Period Changes of RR Lyrae Stars in M68

\begin{tabular}{|c|c|c|c|c|}
\hline Star & $\begin{array}{c}\text { Period } \\
(1950-1953)\end{array}$ & $\begin{array}{c}\text { Period } \\
(1986-1991)\end{array}$ & $\begin{array}{c}\Delta P \times 10^{6} \\
\text { (days) }\end{array}$ & $\begin{array}{c}\alpha \text { (Cycles } \\
\left.\text { per } 10^{6} \mathrm{yr}\right)\end{array}$ \\
\hline V1. & 0.349605 & 0.349574 & $-31 \pm 6$ & $-2.16 \pm 0.42$ \\
\hline V2. & 0.578170 & 0.578185 & $+15 \pm 18$ & $+0.63 \pm 0.76$ \\
\hline V3. & 0.390745 & 0.390734 & $-11 \pm 8$ & $-0.69 \pm 0.50$ \\
\hline V4. & 0.395930 & 0.395940 & $+10 \pm 8$ & $+0.62 \pm 0.49$ \\
\hline V5 ......... & 0.282118 & 0.282117 & $-1 \pm 4$ & $-0.09 \pm 0.35$ \\
\hline V6 ......... & 0.368495 & 0.368493 & $-2 \pm 6$ & $-0.13 \pm 0.40$ \\
\hline V7 ......... & 0.387955 & 0.387958 & $-3 \pm 7$ & $-0.19 \pm 0.44$ \\
\hline V8 $\ldots \ldots \ldots$ & 0.390405 & 0.390407 & $+2 \pm 7$ & $+0.12 \pm 0.44$ \\
\hline V11 ....... & 0.364925 & 0.364931 & $+6 \pm 6$ & $+0.40 \pm 0.40$ \\
\hline V13 ....... & 0.361735 & 0.361742 & $+7 \pm 6$ & $+0.47 \pm 0.40$ \\
\hline V14 & 0.556780 & 0.556803 & $+23 \pm 13$ & $+1.03 \pm 0.57$ \\
\hline V16 ....... & 0.381960 & 0.381960 & $0 \pm 7$ & $0.00 \pm 0.45$ \\
\hline V18 ........ & 0.367340 & 0.367348 & $+8 \pm 6$ & $+0.53 \pm 0.40$ \\
\hline V20 ....... & 0.385775 & 0.385794 & $+19 \pm 7$ & $+1.20 \pm 0.44$ \\
\hline V22 ....... & 0.563440 & 0.563439 & $-1 \pm 15$ & $-0.04 \pm 0.65$ \\
\hline V23 & 0.658868 & 0.658884 & $+16 \pm 16$ & $+0.61 \pm 0.61$ \\
\hline V24 $\ldots \ldots \ldots$ & 0.376485 & 0.376473 & $-12 \pm 7$ & $-0.78 \pm 0.45$ \\
\hline V25 ....... & 0.641520 & 0.641490 & $-30 \pm 30$ & $-1.17 \pm 1.17$ \\
\hline V26 ........ & 0.407040 & 0.407031 & $-9 \pm 8$ & $-0.54 \pm 0.48$ \\
\hline V28 $\ldots \ldots \ldots$ & 0.606767 & 0.606780 & $+13 \pm 9$ & $+0.54 \pm 0.37$ \\
\hline V29 & 0.395240 & 0.395236 & $-4 \pm 8$ & $-0.25 \pm 0.49$ \\
\hline V30 ........ & 0.733650 & 0.733635 & $-15 \pm 27$ & $-0.49 \pm 0.90$ \\
\hline V31 ....... & 0.399660 & 0.399655 & $-5 \pm 8$ & $-0.31 \pm 0.49$ \\
\hline
\end{tabular}


phase shift of the light curve versus time, for each star. then, if the period changed linearly with time, a parabola was fitted through the points and the rate of change derived from coeffiI cients of the parabolic equation. Of the 38 stars in their sample, 16 showed period increases, three showed decreases, three showed both increases and decreases, 10 had constant periods, and for the remaining six stars, the nature of the period changes was uncertain. Because of the long interval over which the M15 observations were made, Smith \& Sandage were able to detect period changes as small as $\alpha=0.09$ cycles per million years for the RRc,d stars and 0.17 for the RRab stars. Most of the period changes they found were at rates smaller than $\alpha=0.4$, the minimum error for our M68 data. Only five of the 38 stars in their sample showed changes larger than $\alpha=0.4$, four increasing and one decreasing. The $O-C$ diagrams for these stars with the large values of $\alpha$ indicate that abrupt period changes have occurred over the interval of the observations. We assume that this is also the reason for the large period changes that we have detected for V1 and V20 in M68. Thus the available data show no evidence for significant differences in the period change behavior of the RR Lyrae variables in these two clusters.

Recent theoretical studies of RR Lyrae stars by Lee (1991) indicate that the mean rate of period change of RR Lyrae stars in globular clusters should depend on horizontal branch morphology. For clusters with extremely blue horizontal branches, he predicts a mean rate $\beta=0.15$ days per million years, and for clusters with red horizontal branches, the rate is smaller. This correlation with HB type occurs because RR Lyrae stars in clusters with blue horizontal branches are more evolved than those in clusters with red horizontal branches according to Lee, Demarque, \& Zinn (1990) and stars near the ZAHB have small period changes (in the mean zero), while more evolved stars have period increases of up to 0.5 days per million years. Lee (1991) has argued that published period changes for the RR Lyrae stars in the five clusters, M3, NGC 6934, M5, M15, and $\omega$ Centauri support this hypothesis. A recent study of 46 RR Lyrae variables in NGC 7006 (Wehlau et al. 1992) indicates that the period changes in this cluster deviate somewhat from Lee's predicted relationship, but not enough to be considered in conflict with his models. Unfortunately, the existing data for M68 are not suitable for testing Lee's theory. More observations of the variables are necessary to give a longer time span so that smaller period changes may be detected and, in addition, further observations are required to establish the morphology of the horizontal branch which is very uncertain (Lee 1990).

We would like to thank two of the resident astronomers of the University of Toronto Southern Observatory for taking some of the plates: Ian Shelton in 1987 July and Jorge GarciaYus in 1989. Also, we thank Denise Giguere for her assistance with the "period change" analysis. We also thank the referee for suggesting that we comment on the earlier observations of V33. One of us (C.M.C.) is grateful for financial support from the Natural Sciences and Engineering Research Council of Canada, and N.R.S. for support under NASA Astrophysics Theory Program.

\section{REFERENCES}

Andrews, P. J. 1979 , QJRAS, 20, 44 1980, in IAU Symp. 85, Star Clusters, ed. J. E. Hesser (Dordrecht: Reidel), 425

Belserene, E. P. 1964, AJ, 69, 475

Clement, C. M. 1990, AJ. 99, 240

Clement, C. M., Jankulak, M. \& Simon, N. R. 1992, ApJ, 395, 192 (CJS)

Clement, C. M., Kinman, T. D., \& Suntzeff, N. B. 1991, ApJ, 372, 273

Clement, C. M., \& Nemec, J. 1990, JRASC, 84, 434

Clement, C. M., Nemec, J. M., Robert, N., Wells, T., Dickens, R. J., \& Bingham, E. A. 1986, AJ, 92,825

Clement, C. M., \& Walker, I. 1990, AJ, 101, 1352

Cox, A. N. 1991, ApJ, 381, L71

Cox, A. N., Hodson, S. W., \& Clancy, S. P. 1983, ApJ, 266, 94

Diaconis, P., \& Efron, B. 1983, Sci. Am., 248, No. 5, 116

Jerzykiewicz, M. Schult, R. H., \& Wenzel, W. 1982, Acta Astron., 32, 357

Kovács, G., Buchler, J. R., \& Marom, A. 1991, A\&A, 252, L27

Kovács, G., Buchler, J. R., Marom, A., Iglesias, C. A., \& Rogers, F. J. 1992, A\&A, 259, L46

Kurtz, D. W. 1985, MNRAS, 213, 773

Lee, Y.-W. 1990, ApJ, 363, 159

Lee, Y.-W., Demarque, P., \& Zinn, R. 1990, ApJ, 350, 155
Lomb, N. R. 1976, Ap\&SS, 1976, 39, 447

McClure, R.D., Vandenberg, D. A., Bell, R. A., Hesser, J. E., \& Stetson, P. B. 1987, AJ, 93, 1144

Mendes de Oliveira, C., \& Smith, H. 1990, PASP, 102, 652

Nemec, J. M. 1985, AJ, 90, 240

Nemec, J. M., \& Clement, C. M. 1989, AJ, 98, 860

Petersen, J. O. 1973, A\&A, 27, 89

. 1984, A\&A, 139, 496

Press, W. H., \& Rybicki, G. B. 1989, ApJ, 338, 277

Rogers, F. J., \& Iglesias, C. A. 1992, ApJS, 79, 507

Rosino, L., \& Pietra, S. 1954, Bologna Publ., 6 No. 5

Sawyer Hogg, H. 1973, Publ. David Dunlap Obs., 3, No. 6

Scargle, J. D. 1982, ApJ, 263, 835

Simon, N. R. 1979, A\&A, 74, 30

Simon, N. R., \& Teays, T. J. 1982, ApJ, 261, 586

Smith, H. A., \& Sandage, A. 1981, AJ, 86, 1870

Stellingwerf, R. F. 1978, ApJ, 224, 953

Stobie, R. R. 1970, Observatory, 90, 20

Suntzeff, N. B., Kinman, T. D., \& Kraft, R. P. 1991, ApJ, 367, 528

van Agt, S. L. Th. J. \& Oosterhoff, P. Th. 1959, Leiden Ann, 21, Pt. 4,253

Wehlau, A., Nemec, J. M., Hanlan, P., \& Rich, R. M. 1992, AJ, 103, 1583 